\section{Radiotherapy of Ocular Disease}

H erausgeber: T. Wiegel, N. B ornfeld, M .H . Förster, W. H inkelbein (B erlin)

Front R adiat Ther O ncol. B asel, Karger vol 30, 1997, 302 S., 263,- D M

ISB N 3-8055-6456-2

Mit dem jetzt erschienenen Buch wird der aktuelle wissenschaftliche Stand der Strahlentherapie von A ugenerkrankungen dokumentiert. Es werden sämtliche heutigen A spekte dieser $B$ ehandlungsform beleuchtet. $\mathrm{H}$ ierbei ist es zunächst zu begrüßen, daß in zwei speziellen Aufsätzen auf die magnetresonanztomographische Bildgebung in bezug auf $A$ ugentumoren eingegangen wird. Weitere vier A rtikel beschäftigen sich mit den grundlegenden radiotherapeutischen Techniken: D osimetrie und A nwendung von radioaktiv belegten A ugenschalen, stereotaktische Bestrahlung sowie Brachytherapie. $D$ ie weiteren $A$ bschnitte des $B$ uches sind den einzelnen $E r$ krankungen gewidmet: $\mathrm{R}$ etinoblastom, $\mathrm{M}$ elanom, choroideale $M$ etastasen, orbitale Lymphome und andere Tumoren, endokrine Ophthalmopathien und gutartige Erkrankungen ( $z$.B. $M$ akula-D egeneration). $Z$ wei abschließende Artikel stellen die $\mathrm{N}$ ebenwirkungen und Spätfolgen bei der $\mathrm{B}$ ehandlung von A ugenerkrankungen mit den eingangs genannten $M$ ethoden dar.

D er umfangreiche Inhalt wird auch durch praktische B eispiele aus der dreidimensionalen $B$ estrahlungsplanung illustriert, oft in Farbe, so daß dem klinisch tätigen R adiotherapeuten eine wesentliche 0 rientierungshilfe gegeben ist.

D ieses Buch ist für alle $\mathrm{K}$ liniker interessant, die sich mit der Behandlung gutartiger und bösartiger Augenprozesse befassen, darüber hinaus als E rgänzung für die $M$ itarbeiter in Weiterbildung geeignet und sicher auch für $O$ phthalmologen von großem Interesse.

$M$. B usch, M ünchen

\section{Lehrbuch der Palliativmedizin}

H erausgeber: E. A ulbert, D. Zech

Stuttgart, Schattauer, 1997, 1043 S., 248,- D M

ISB N 3-7945-1708-3

M it diesem ausgezeichneten Lehrbuch und $\mathrm{N}$ achschlagewerk zur Palliativmedizin ist nun endlich ein weiterer Schritt in Richtung einer E tablierung dieser, nicht nur in Forschung und Lehre oft gemiedenen, relativ jungen Fachrichtung gelungen. R obert Twycross, einer der «U rväter» der Palliativmedizin, betont in seinem Vorwort, daß Palliativmedizin keineswegs als «A bstellkammer für hoffnungslose Fälle» gesehen werden darf. M ehr als zwei D rittel aller Tumorpatienten und fast alle, die unter einer chronisch progredienten, nicht mehr heilbaren
E rkrankung leiden, würden von einer begleitenden palliativmedizinischen $B$ ehandlung profitieren. Palliativmedizin beinhaltet nicht nur die ganzheitliche Versorgung von Patienten mit unheilbaren E rkrankungen, sondern versucht auch, die Bedürfnisse der betroffenen Familien vor und nach dem Tod des Patienten zu berücksichtigen. Nicht die Verlängerung der Überlebenszeit um jeden Preis, sondern die Erhaltung und Verbesserung von Lebensqualität mit Berücksichtigung menschlicher K ernbedürfnisse in der letzten Lebensphase sind $\mathrm{H}$ auptziele einer palliativmedizinisch orientierten, interdisziplinären Behandlung. B ei der Lektüre dieses Buches wird schnell klar, daß eine Veränderung unseres K rankheitsverständnisses und unserer beruflichen Praxis Voraussetzung dafür sein wird, daß solche B ehandlungskonzepte $E$ ingang in die moderne $\mathrm{H}$ ochleistungsmedizin finden.

$D$ as Lehrbuch gliedert sich in übersichtlicher Form in die A bschnitte Grundlagen und A nwendungsbereiche der Palliativmedizin, interdisziplinäre Behandlungsformen, K rebsschmerzbehandlung, allgemeine Symptomkontrolle, Pflege, psychologische Begleitung, Rehabilitation, Sozialrecht, Personalentlastung sowie D okumentation und Q ualitätskontrolle. Im A nhang finden sich eine Liste der stationären Palliativeinrichtungen und $\mathrm{H}$ ospize sowie ein A uszug aus der B etäubungsmittel-Verschreibungsverordnung.

Unter den über hundert Autoren befinden sich nicht nur Mediziner fast aller Fachrichtungen, Vertreter von Pflege, Selbsthilfeorganisationen, $\mathrm{H}$ ospiz- und Palliativeinrichtungen, sondern neben vielen anderen auch betroffene Patienten, Physio- und E rgotherapeuten, Sozialarbeiter, M usiktherapeuten, Theologen und E thiker, Psychologen und Soziologen.

B etrachtet man sich diese L iste, so wird schnell kar, wie facettenreich eine Behandlung auch in fortgeschrittenen K rankheitsstadien sein kann, insbesondere dann, wenn die Möglichkeiten einer interdisziplinären Z usammenarbeit genutzt werden. $D$ as $L$ ehrbuch gibt umfassende $H$ ilfestellung für die B ehandlung in der Phase einer $\mathrm{K}$ rankheit, in der man gemeinhin glaubt, nichts mehr tun zu können.

D as K apitel «E thische Fragen in der Palliativmedizin» dürfte den Leser in besonderem $M$ aße beeindrucken. Spätestens hier, aber auch in einigen $\mathrm{K}$ apiteln des $\mathrm{A}$ bschnitts «Psychologische Begleitung» wird klar, daß es letztlich um K ernfragen menschlichen L ebens geht.

In über 500 Seiten wird auf B ehandlung von Symptomen wie Schmerz, K achexie, Ü belkeit und A temnot eingegangen und es werden neben palliativ internistisch-onkologischen, chirurgischen und radiologischen Therapien weitere B ehandlungsmöglichkeiten aufgezeigt. E rfahrungen und E rkenntnisse erfahrener Palliativmediziner und Schmerztherapeuten schlagen sich in Therapieempfehlungen nieder, die praxisnah und umsetzbar sind. D ie weit verbreitete 0 pioidphobie wird mehrfach als großes $\mathrm{H}$ indernis für die Durchführung einer adäquaten Schmerztherapie genannt. In den K apiteln der interventionell und invasiv arbeitenden Kollegen wird klar zwischen dem
KARGER (c) 1998 S. Karger $\mathrm{G} \mathrm{mbH}$, Freiburg

Fax (0761) 4520714

www.karger.com 
«M achbaren» und dem hinsichtlich Lebensqualität «E mpfehlenswerten» unterschieden.

Das Buch ist durch das ausführliche Sachverzeichnis auch als Nachschlagewerk geeignet. Die Beiträge der einzelnen A utoren sind in der R egel inhaltlich und formal so klar strukturiert, daß auf die zahlreichen fettgedruckten Hervorhebungen hätte verzichtet werden können.

Palliativmedizin gehört neben Prävention, Früherkennung und kurativer B ehandlung für die Weltgesundheitsorganisation zu den essentiellen Komponenten eines umfassenden Tumorbehandlungsplanes. $\mathrm{D}$ aß dieser Bereich jedoch immer noch zu den Stiefkindern der Medizin zählt, mag verschiedene U $r$ - sachen haben. Einerseits vielleicht eine gewisse A bneigung, sich mit dem Thema Tod und Sterben auseinanderzusetzen, andererseits aber auch möglicherweise die Auffassung, daß Forschung im B ereich palliativmedizinischer Themen nur ein geringes wissenschaftliches A nsehen genießen könnte.

D ie Herausgeber des Buches und viele der Autoren zählen zweifelsohne zu denjenigen, die Forschung und L ehre auch in diesem Bereich vorangetrieben haben. Ich wünsche diesem ersten deutschsprachigen L ehrbuch der Palliativmedizin viele L eser und hoffe, daß es dazu beiträgt, Verbreitung und A kzeptanz palliativmedizinischer B ehandlung zu fördern.

Carla Weber, M annheim 